\title{
Rotigotine in Combination with the MAO-B Inhibitor Selegiline in Early Parkinson's Disease: A Post Hoc Analysis
}

\author{
Nir Giladi ${ }^{\mathrm{a}, *}$, Mahnaz Asgharnejad ${ }^{\mathrm{b}}$, Lars Bauer $^{\mathrm{c}}$, Frank Grieger ${ }^{\mathrm{c}}$ and Babak Boroojerdi ${ }^{\mathrm{c}}$ \\ ${ }^{a}$ Chairman of the Neurological Institute, Tel Aviv Medical Center, Director of the Department of Neurology and \\ Neurosurgery, Sackler School of Medicine, Sagol School of Neuroscience, Tel Aviv University, Tel Aviv, Israel \\ ${ }^{\mathrm{b}}$ UCB Pharma, Raleigh, NC, USA \\ ${ }^{\mathrm{c}}$ UCB Pharma, Monheim am Rhein, Germany
}

Accepted 4 March 2016

\begin{abstract}
.
Background: Monoamine oxidase B (MAO-B) inhibitors and dopamine receptor agonists are common first-line treatment options in early Parkinson's disease (PD).

Objective: To evaluate the efficacy and safety of rotigotine transdermal patch as an add-on therapy to an MAO-B inhibitor in patients with early-PD.

Methods: In two Phase III, randomized, double-blind, placebo-controlled studies in early-PD (SP512, SP513), patients were randomized to rotigotine (titrated to optimal dose $\leq 8 \mathrm{mg} / 24 \mathrm{~h}$ ) or placebo, and maintained for 24 (SP512) or 33 (SP513) weeks. Post hoc analyses were performed on pooled data for patients receiving an MAO-B inhibitor (selegiline) at a stable dose at randomization and throughout the studies, with groups defined as "Selegiline+Rotigotine" and "Selegiline+Placebo". Outcome measures included change from baseline in Unified Parkinson's Disease Rating Scale (UPDRS) II (activities of daily living), III (motor), UPDRS II+III and responders (patients achieving $\geq 20 \%, \geq 25 \%$ or $\geq 30 \%$ decrease in UPDRS II+III). As post hoc analyses, $p$-values are exploratory.

Results: 130 patients were evaluable for efficacy analyses ("Selegiline+Rotigotine": 84, "Selegiline+Placebo": 46). Combined treatment with rotigotine and selegiline improved UPDRS III and UPDRS II+III scores versus selegiline alone (LS-mean [95\% CI] treatment difference for UPDRS III: -4.89 [-7.87 to -1.91 ], $p=0.0015$; for UPDRS II+III: -5.76 [ -9.71 to -1.82 ], $p=0.0045$ ). Higher proportion of patients in the "Selegiline+Rotigotine" group were classified as $\geq 20 \%, \geq 25 \%$ or $\geq 30 \%$ responders (all $p<0.001$ ). Combined treatment appeared more effective in patients aged $\leq 65$ years versus $>65$ years (although patient numbers in the subgroups were low). Adverse event profile was consistent with the known safety profile of rotigotine. Conclusions: In these post hoc analyses, adjunctive treatment with rotigotine in patients already receiving an MAO-B inhibitor improved UPDRS II+III score; this appeared to be largely driven by improvements in the motor aspects of PD.
\end{abstract}

Keywords: Dopamine receptor agonist, monoamine oxidase inhibitors, Parkinson's disease, clinical trial

\section{INTRODUCTION}

*Correspondence to: Nir Giladi, Neurological Institute, Tel Aviv Medical Center, 6 Weizmann Street, 64239 Tel Aviv, Israel. Tel.: +972 36974790; Fax: +972 36974153; E-mail: nirg@tlvmc.gov.il.
Initial medication choice in patients with earlystage Parkinson's disease (PD), as well as subsequent therapy adjustments, are highly individualized and 
depend on a number of factors relating both to the drug (e.g., efficacy, safety profile) and to the patient (e.g., age, symptom characteristics/severity) [1-3]. Patients are commonly treated with monoamine oxidase B (MAO-B) inhibitors or dopamine receptor agonists (DAs) as first-line therapy in the early stages of the disease $[1,2,4]$, which may delay the time to initiation with levodopa therapy and the incidence of motor fluctuations [1, 4-6]. Delaying levodopa may be particularly important for younger patients who are more prone to levodopa-induced motor complications with long-term use $[1,2]$.

Although there are no published head-to-head, double-blind, randomized, controlled studies that directly compare the relative efficacy of MAO-B inhibitors versus DAs, indirect comparisons show that improvements in Unified Parkinson's Disease Rating Scale (UPDRS) scores are usually larger with DAs than those reported with MAO-B inhibitors, suggesting a greater symptomatic effect with the agonists $[1,7]$. Moreover, a systematic review demonstrated that whilst there was no difference in the reduction of motor fluctuations between patients receiving MAO$\mathrm{B}$ inhibitors and patients receiving DAs, patients receiving MAO-B inhibitors were more likely to require add-on therapy during the follow-up period of at least 1 year [7]. Of note, in a recent open-label, randomized study of newly diagnosed patients with $\mathrm{PD}, \mathrm{MAO}-\mathrm{B}$ inhibitors as initial levodopa-sparing therapy were suggested to be at least as effective as DAs when assessing patient-rated mobility scores [8]. Regarding safety, although both MAO-B inhibitors and DAs can induce dopaminergic adverse reactions, MAO-B inhibitors may have a milder adverse event (AE) profile $[1,2,9,10]$; however, comparisons of the MAO-B inhibitors and the newer non-ergot-derived DAs are not available [7]. Generally, in patients with mild symptoms, an MAO-B inhibitor may be recommended before moving on to a DA or levodopa as the disease progresses $[2,10]$. In addition, a recent review on treatment optimization in early-stage PD has suggested that using a combination of drugs rather than a single drug at high dose may be more or equally effective, and may potentially minimize the risk of side effects [3].

Two irreversible MAO-B inhibitors, selegiline and rasagiline, are currently available for the symptomatic treatment of early-stage PD [11]; they act to reduce striatal metabolism of dopamine, leading to greater dopamine availability $[1,11]$. Selegiline was the first MAO-B inhibitor to be used for the treatment of PD, and a number of studies have demonstrated that selegiline monotherapy provides a small symptomatic benefit in early-stage PD [12-17]. Rotigotine is a non-ergot-derived DA with activity across D1 through D5 receptors as well as select adrenergic and serotonergic sites [18]; continuous transdermal delivery of rotigotine maintains stable plasma levels over 24 hours with a single daily application [19]. The efficacy of rotigotine transdermal patch versus placebo in patients with early-stage PD has been established in two pivotal 6-month, randomized, double-blind, placebo-controlled studies (SP512 and SP513); rotigotine was associated with a statistically significant benefit compared with placebo on measures of motor function and activities of daily living, as assessed by the UPDRS II+III total scores [20-22]. In these studies, treatment with a concomitant MAO-B inhibitor was permitted.

The objective of the current post hoc analyses of the SP512 and SP513 studies was to evaluate the potential added value of concomitant treatment with rotigotine transdermal patch in patients with early-stage PD who were already receiving an MAO-B inhibitor at study entry. We also assessed the safety and tolerability of this concomitant therapy with rotigotine versus patients receiving MAO-B inhibitor monotherapy. Post hoc analyses of pooled data from the SP512 and SP513 studies are reported here.

\section{METHODS}

\section{SP512 and SP513 study design and patients}

The SP512 [21, 22] and SP513 [20] studies were Phase III, multicenter, randomized, double-blind, controlled, parallel-group clinical studies of rotigotine transdermal patch in patients with early-stage PD. Eligible patients were 30 years of age or older, had early-stage idiopathic PD of up to 5 years in duration, had UPDRS III score $\geq 10$ and were Hoehn and Yahr stage 1-3 (mild to moderate PD). Treatment with other DAs or levodopa was not permitted during the studies or in the 1 month prior to enrollment. Treatment with MAO-B inhibitors was allowed during the study, provided the dose had been stable for 28 days prior to study baseline, and remained stable throughout the duration of the study. Full inclusion and exclusion criteria, including permitted concomitant medications, are published [20-22].

In SP512, 277 patients were randomized 2:1 to receive a transdermally delivered rotigotine (titrated to optimal effective dose of $2-6 \mathrm{mg} / 24 \mathrm{~h}$ ) or placebo 
during a 24-week maintenance phase. In SP513, 561 patients were randomized $2: 2: 1$ to receive transdermally delivered rotigotine (titrated to optimal effective dose of $2-8 \mathrm{mg} / 24 \mathrm{~h}$ ), oral ropinirole (titrated to optimal effective dose of $0.75-24 \mathrm{mg} /$ day) or placebo; maintenance phase for rotigotine was 33 weeks.

Efficacy in SP512 and SP513 studies was assessed by the absolute change in UPDRS II+III total scores from baseline to end of maintenance (EoM) phase (primary variable), absolute change in UPDRS II (activities of daily living) subscore and UPDRS III (motor) subscore and UPDRS 20\% responder analyses (secondary outcomes).

The studies were conducted in accordance with the laws of the countries involved, Good Clinical Practice and the Declaration of Helsinki. The study protocols, amendments and patient informed consents were reviewed by national, regional or investigational site ethics committees or institutional review boards.

\section{Post hoc analyses of patients receiving an MAO-B inhibitor}

\section{Patients included in post hoc analyses}

In these post hoc analyses of the SP512 and SP513 studies, data are reported for patients who were receiving concomitant treatment with an MAO-B inhibitor at randomization, and were evaluable for efficacy (i.e., met the full analysis set criteria). All patients who were receiving an MAO-B inhibitor were on selegiline, as rasagiline was not yet available at the time the studies were conducted. Patients who received selegiline and concomitant placebo are referred to as the "Selegiline+Placebo" group, and those who received selegiline and concomitant rotigotine are referred to as the "Selegiline+Rotigotine" group.

\section{Post hoc analyses: Efficacy}

Efficacy was assessed by the absolute change from baseline to EoM in UPDRS II+III total scores, UPDRS III (motor) subscore, UPDRS II (ADL) subscore and proportion of patients achieving $\geq 20 \%$, $\geq 25 \%$ or $\geq 30 \%$ decrease in UPDRS II+III total score (responder analysis). For these outcomes, subgroup analyses by age at study baseline also were performed: patients $\leq 65$ years (younger patients) and patients $>65$ years (older patients). In addition, to account for differences in baseline UPDRS scores between the age groups, an analysis of the percentage change of the mean in the UPDRS scores from baseline to EoM also was performed for the age groups.

\section{Post hoc analyses: Safety and tolerability}

Safety and tolerability assessments included incidence of AEs and discontinuations due to AEs. In addition, considering that both MAO-B inhibitors and DAs can induce orthostatic hypotension [23], analyses were performed to assess possible worsening of the hypotensive effect with combined therapy. For the assessment of orthostatic hypotension, the following MedDRA Preferred Terms associated with orthostatic hypotension were available: "postural hypotension" (included the investigatorreported terms "symptomatic orthostatic hypotension", "asymptomatic orthostatic hypotension", "clinically significant postural hypotension" and "postural hypotension"), "hypotension" (included "symptomatic hypotension" and "hypotension") and "syncope" (included "brief loss of consciousness", "diaphoresis/nausea/vagovagal episode", "fainted", "loss of consciousness" and "syncope"). In addition, vital sign data on orthostatic hypotension were analyzed according to: (1) protocol-specified criteria, defined as a persistent decrease of $\geq 20 \mathrm{~mm} \mathrm{Hg}$ in systolic blood pressure (SBP) or of $\geq 10 \mathrm{~mm} \mathrm{Hg}$ in diastolic blood pressure (DBP) upon standing; and (2) US Food and Drug Administration (FDA)-specified criteria, defined as a SBP decrease of $\geq 40 \mathrm{~mm} \mathrm{Hg}$ or DBP decrease of $\geq 20 \mathrm{~mm} \mathrm{Hg}$ upon standing.

\section{Statistical analyses}

Efficacy analyses were performed on the full analysis set (patients with baseline and at least one post-baseline UPDRS assessment) using last observation carried forward approach. Safety analyses were performed on the safety set (all patients who received at least one dose of study medication [placebo or rotigotine]). The "Selegiline+Placebo" versus "Selegiline+Rotigotine" treatment difference for the change from baseline to EoM (absolute and percent changes) in UPDRS scores were assessed using an analysis of covariance model with treatment and (pooled) site as factors and baseline UPDRS scores as the covariate. The UPDRS 20\%, 25\% and $30 \%$ responder rates were analyzed using asymptotic normal approximation methodology. As these are post hoc analyses, all statistical testing is considered exploratory and $p$-values $<0.05$ do not infer statistical significance. 


\section{RESULTS}

\section{Patients}

Of the 610 patients who were randomized in the SP512 and SP513 studies to rotigotine or placebo, 603 were included in the full analysis set; 130/603 $(21.6 \%)$ patients were receiving treatment with selegiline at stable dose at randomization and are included in the current analyses ("Selegiline+Placebo": 46; "Selegiline+Rotigotine": 84). In total, $107 / 130$ (82.3\%) patients completed the study ("Selegiline+Placebo": 39 [84.8\%]; "Selegiline+Rotigotine": 68 [81.0\%]); the most common reason for premature discontinuation was AEs ("Selegiline+Placebo": 3 [6.5\%]; "Selegiline+Rotigotine": 9 [10.7\%]), followed by withdrawal of consent (4 [8.7\%]; 4 [4.8\%]), lack of efficacy $(4[8.7 \%] ; 3[3.6 \%])$, unsatisfactory compliance $(0 ; 1[1.2 \%])$ and other $(0 ; 1[1.2 \%])$.

The demographics and baseline characteristics of the pooled study population, including mean selegiline dose, were similar between the treatment groups (Table 1), with the exception of gender: $76 \%$ of the "Selegiline+Placebo" patients and $56 \%$ of the "Selegiline+Rotigotine" patients were male. The mean \pm standard deviation rotigotine dose during the maintenance phase was $16.2 \pm 2.80$ (median: 18.0; range: $4.5-18.0) \mathrm{mg} / 24 \mathrm{~h}$.

\section{Efficacy}

\section{Change from baseline to EoM in UPDRS scores}

All patients. Absolute change from baseline to EoM in mean UPDRS II+III total score numerically improved in the "Selegiline+Rotigotine" group compared with the "Selegiline+Placebo" group; least squares (LS) mean (95\% confidence interval [CI]) treatment difference: $-5.76(-9.71$ to -1.82$)$, $p=0.0045$ (Fig. 1A). Absolute change from baseline to EoM in mean UPDRS III subscores also numerically improved in patients treated with rotigotine and selegiline compared with those treated with selegiline alone; LS mean $(95 \% \mathrm{CI})$ treatment difference: -4.89 ( -7.87 to -1.91$), p=0.0015$ (Fig. 1B). UPDRS II scores indicated a small numerical improvement in the "Selegiline+Rotigotine" group; LS mean (95\% CI) treatment difference: -0.88 ( -2.23 to 0.46$)$; $p=0.1965$ ) (Fig. 1C).

Analysis by age: patients $\leq 65$ years

and $>65$ years. A small numerical difference in baseline UPDRS scores was observed between older and younger patients; the older patient subgroup (i.e., patients $>65$ years of age at study baseline) had higher (i.e., worse) mean UPDRS scores at baseline compared with the younger patients (Fig. 2).

In patients $\leq 65$ years of age, absolute changes in all mean UPDRS scores indicated numerical improvements in the "Selegiline+Rotigotine" group compared with the "Selegiline+Placebo" group (Fig. 2). As with the results obtained for all patients, the largest numerical improvements in favor of "Selegiline+Rotigotine" were seen for the UPDRS II+III total score and UPDRS III subscore (Fig. 2A). In patients $>65$ years of age, the differences in the absolute mean UPDRS scores between the "Selegiline+Rotigotine" groups and "Selegiline+Placebo" groups were less evident (Fig. 2B).

As the baseline UPDRS scores differed slightly between the age subgroups, percentage change of the mean from baseline to EoM are also presented. In this analysis, when taking into account baseline scores, treatment with "Selegiline+Rotigotine" resulted in larger percentage improvements in all UPDRS scores than treatment with "Selegiline+Placebo" in both age groups (Fig. 3).

\section{UPDRS II+III responder analysis}

All patients, and analysis by age. For all patients, a higher proportion of patients treated with rotigotine and selegiline compared with patients treated with selegiline alone were classified as $20 \%$, $25 \%$ and $30 \%$ UPDRS II+III responders, with 50/84 (59.5\%) "Selegiline+Rotigotine" patients versus 13/46 (28.3\%) "Selegiline+Placebo" patients achieving at least $20 \%$ reduction in their UPDRS II+III scores $(p=0.0002)$ (Fig. 4A). Higher 20\%, $25 \%$ and $30 \%$ responder rates with "Selegiline+Rotigotine" also were observed in both age subgroups. In patients aged $\leq 65$ years, 32/54 (59.3\%) "Selegiline+Rotigotine" patients versus 9/32 $(28.1 \%)$ "Selegiline+Placebo" patients were classified as $20 \%$ responders $(p=0.0027)$ (Fig. 4B), and in patients $>65$ years of age, 18/30 (60.0\%) "Selegiline+Rotigotine" patients versus 4/14 (28.6\%) "Selegiline+Placebo" patients were classified as $20 \%$ responders ( $p=0.0365)$ (Fig. 4C).

\section{Safety and tolerability}

Overall, 71/84 (85\%) patients receiving "Selegiline+Rotigotine" and 42/46 (91\%) patients receiving 
Table 1

Pooled demographic and baseline characteristics for the SP512 and SP513 studies (safety set)

\begin{tabular}{lcc}
\hline & Selegiline+Placebo $(n=46)$ & Selegiline+Rotigotine $(n=84)$ \\
\hline Age, mean \pm SD (range), years & $59.7 \pm 9.89(37-79)^{\mathrm{a}}$ & $60.6 \pm 9.95(37-77)^{\mathrm{a}}$ \\
$\leq 65$ years, $n$ & 32 & 54 \\
$>65$ years, $n$ & 14 & 30 \\
Male, $n(\%)$ & $35(76)^{\mathrm{a}}$ & $47(56)^{\mathrm{a}}$ \\
Time since diagnosis, mean $\pm \mathrm{SD}$ (range), years & $1.8 \pm 1.50(0-5)$ & $1.8 \pm 1.56(0-5.3)$ \\
Seligiline dose $(\mathrm{mg} /$ day), mean \pm SD & $9.7 \pm 5.68^{\mathrm{b}}$ & $10.3 \pm 10.72$ \\
\hline
\end{tabular}

$\mathrm{SD}=$ standard deviation. ${ }^{\mathrm{a}}$ Enrolled set. ${ }^{\mathrm{b}} n=45$.

\section{A UPDRS II+III}

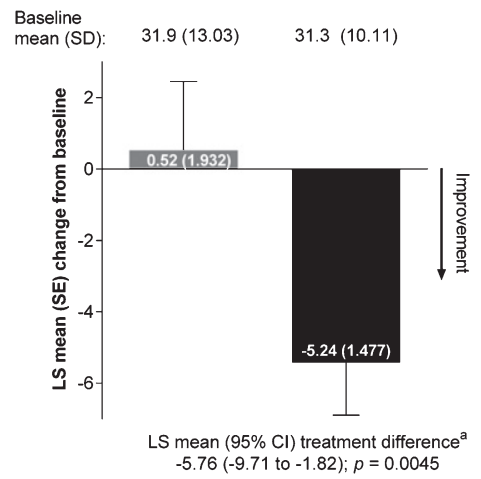

B UPDRS III

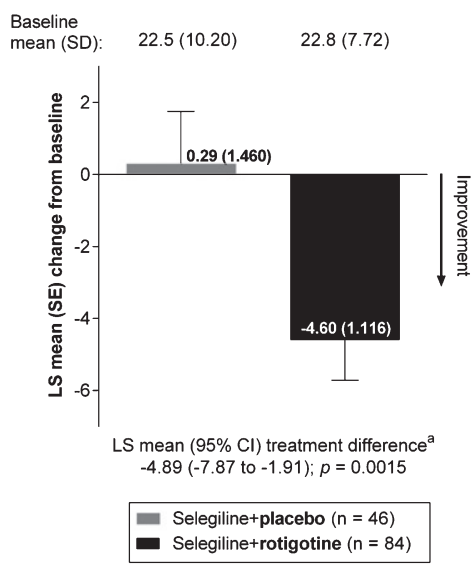

C UPDRS II

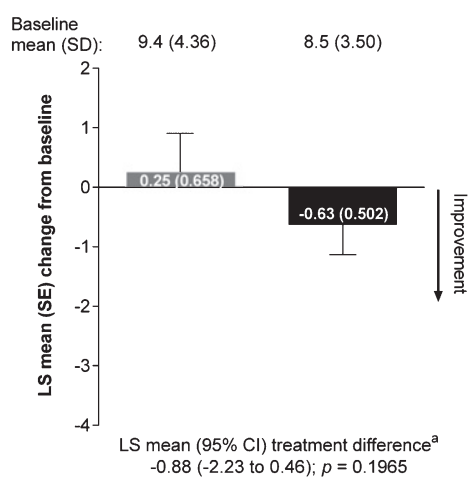

Fig. 1. Absolute LS mean change from baseline to end of maintenance in UPDRS scores (full analysis set, last observation carried forward). $\mathrm{CI}=$ confidence interval; $\mathrm{LS}=$ least squares; $\mathrm{SD}=$ standard deviation; $\mathrm{SE}=$ standard error; UPDRS = Unified Parkinson's Disease Rating Scale. ${ }^{a}$ Analysis of covariance model with treatment and (pooled) site as factors and baseline value as covariate. As post hoc analyses, all $p$-values presented are exploratory.

"Selegiline+Placebo" reported AEs. The incidence of the most common AEs (i.e., application site reactions [ASRs], nausea, somnolence and dizziness) was numerically higher in patients receiving "Selegiline+Rotigotine" (Table 2).

In the analysis of orthostatic hypotension reported as AEs, 1/84 (1\%) patient reported an AE in the "Selegiline+Rotigotine" group ("postural hypotension") and 3/46 (7\%) patients reported AEs in the "Selegiline+Placebo" group ("hypotension" [one patient] and "syncope" [two patients]).

Analysis of vital sign data on orthostatic hypotension as specified by protocol ( $\geq 20 \mathrm{~mm} \mathrm{Hg}$ decrease in SBP or $\geq 10 \mathrm{~mm} \mathrm{Hg}$ decrease in DBP) did not identify any patients as having orthostatic hypotension at study baseline (baseline data missing for one patient in the "Selegiline+Placebo" group); however, 46/130 (35.4\%) patients were identified as having at least one instance of post-baseline orthostatic hypotension. Orthostatic hypotension was less common in patients in the "Selegiline+Rotigotine" group (25/84 [29.8\%]) than in the "Selegiline+Placebo" group
(21/46 [45.7\%]). Analysis by the FDA-specified criteria $(\geq 40 \mathrm{~mm} \mathrm{Hg}$ decrease in SPB or $\geq 20 \mathrm{~mm}$ $\mathrm{Hg}$ decrease in DBP) yielded similar results. Overall, 12/130 (9.2\%) patients discontinued owing to AEs ("Selegiline+Placebo": 3 [6.5\%]; "Selegiline+Rotigotine": 9 [10.7\%]). Of these, five patients discontinued owing to serious AEs ("Selegiline+Placebo": severe increased QT [one patient]; "Selegiline+Rotigotine": mild palpitations [one patient], severe erythematous rash [one patient], severe pruritus and severe ASR [one patient] and moderate ASR [one patient]); all serious AEs leading to discontinuation were considered to be probably or highly probably related to study medication.

\section{DISCUSSION}

In these post hoc analyses of two pivotal doubleblind studies of rotigotine in early-stage PD, the addition of rotigotine in patients already receiving treatment with the MAO-B inhibitor selegiline improved UPDRS scores to a greater extent than in 


\section{A Patients aged $\leq 65$ years}

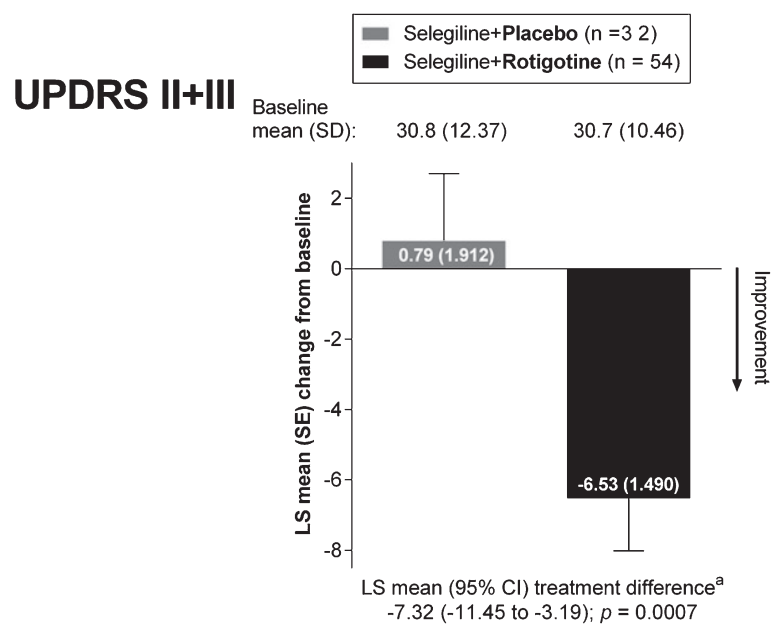

UPDRS III
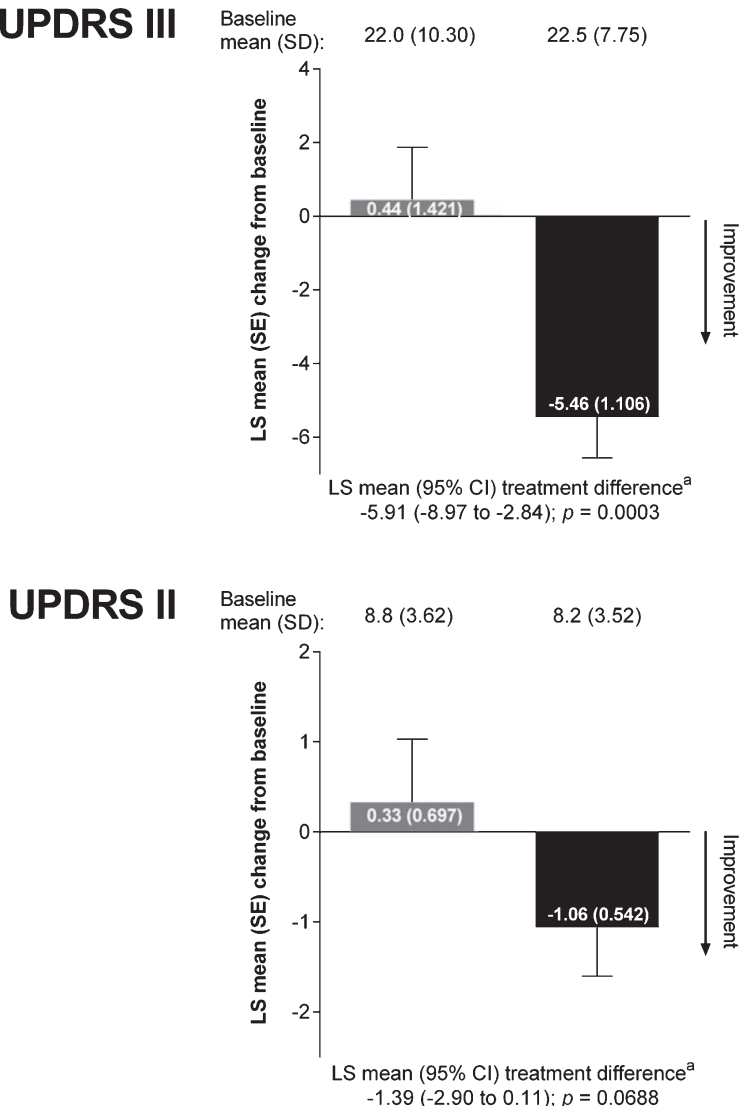

\section{B Patients aged $>65$ years}
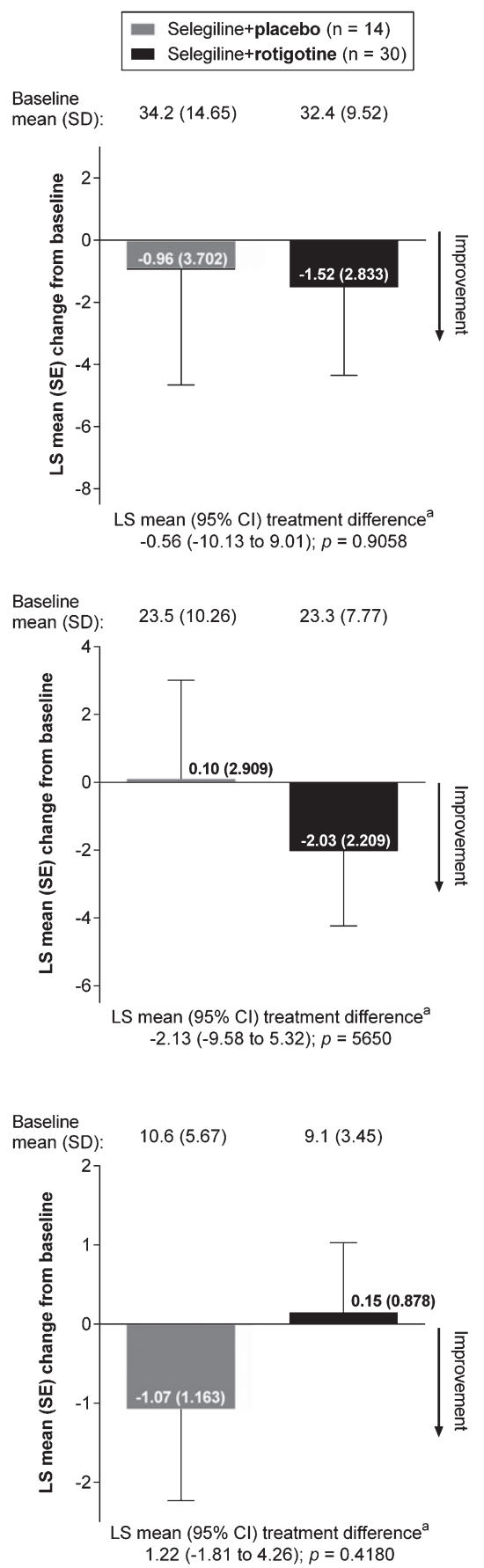

Fig. 2. Absolute LS mean change from baseline to end of maintenance in UPDRS scores, analysis by age (full analysis set, last observation carried forward). $\mathrm{CI}=$ confidence interval; $\mathrm{LS}=$ least squares; $\mathrm{SD}=$ standard deviation; $\mathrm{SE}=$ standard error; UPDRS = Unified Parkinson's Disease Rating Scale. ${ }^{a}$ Analysis of covariance model with treatment and (pooled) site as factors and baseline value as covariate. As post hoc analyses, all $p$-values presented are exploratory. 
A Patients aged $\leq 65$ years

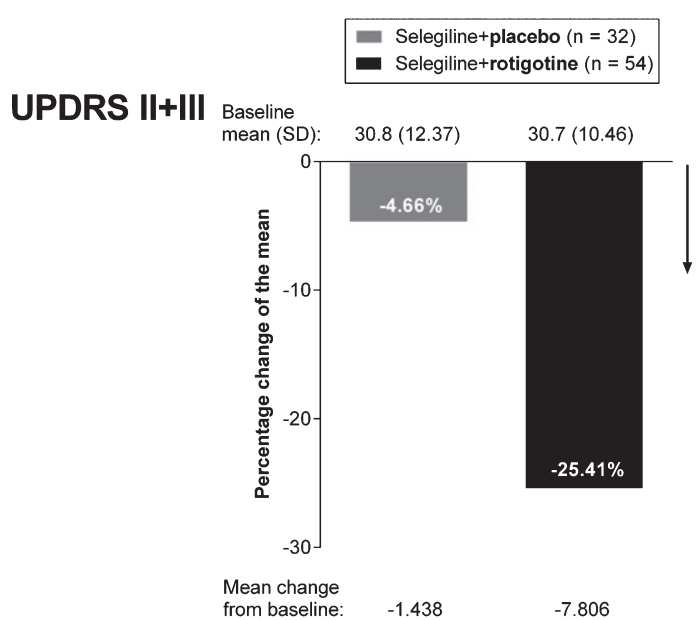

B Patients aged $>65$ years
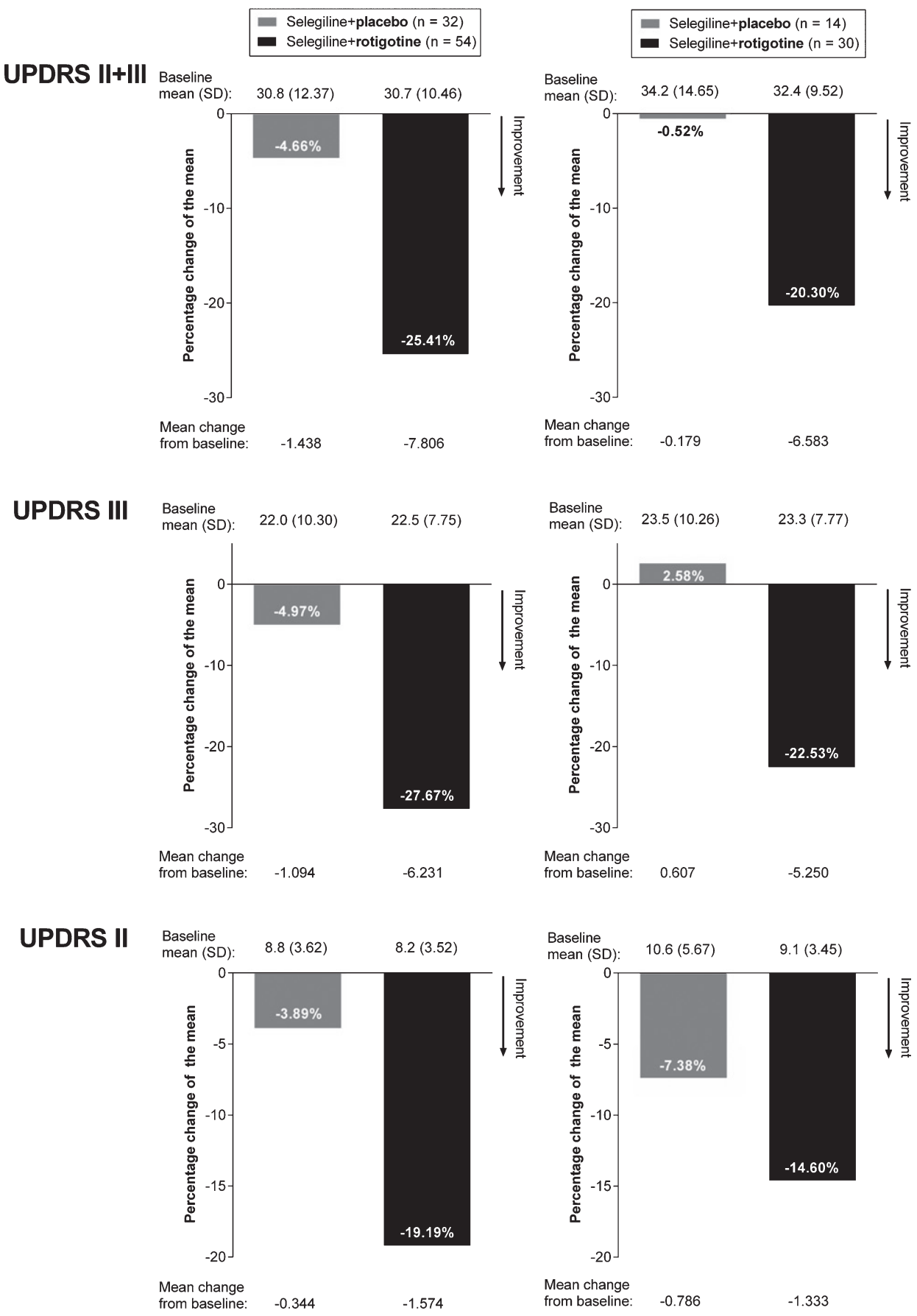

Fig. 3. Percentage change of the mean in UPDRS scores from baseline to end of maintenance, analysis by age (full analysis set, last observation carried forward). SD = standard deviation; UPDRS = Unified Parkinson's Disease Rating Scale.

patients treated with selegiline alone. The improvements were achieved at an acceptable additional expense of AEs, and generally without compromising overall tolerability.
In patients receiving combination therapy ("Selegiline+Rotigotine" group), an improvement was observed in UPDRS II+III total scores $(p<0.005$, exploratory analysis) at EoM compared 
A. All patients

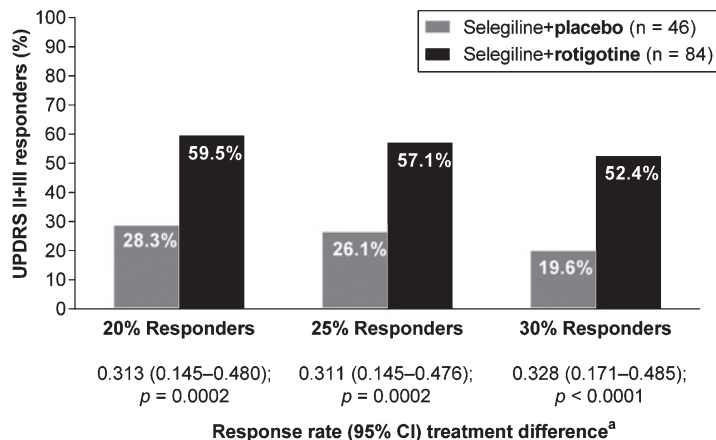

B. $\leq 65$ years

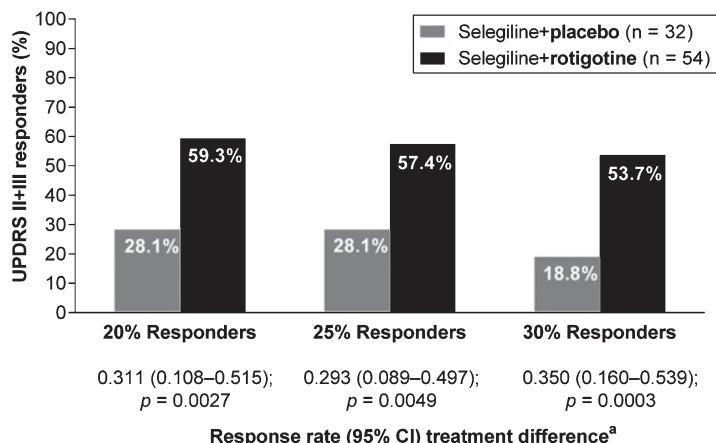

C. $>65$ years

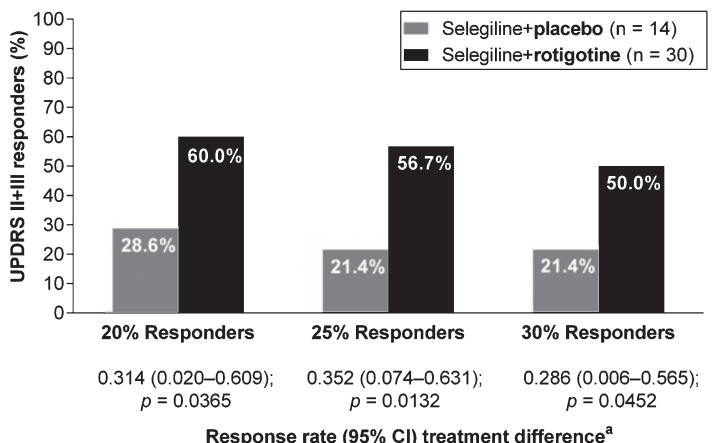

Fig. 4. UPDRS II+III responder analyses (full analysis set, last observation carried forward). $\mathrm{CI}=$ confidence interval; UPDRS = Unified Parkinson's Disease Rating Scale. ${ }^{\text {a }}$ Response rates analyzed using asymptotic normal approximation methodology. As post hoc analyses, all $p$-values presented are exploratory.

with patients receiving selegiline monotherapy ("Selegiline+Placebo" group); in patients receiving selegiline monotherapy, the mean UPDRS II+III scores at EoM were poorer compared with baseline. The improvement in UPDRS II+III scores with combination therapy appeared to be driven largely by improvements in the motor aspects of PD (UPDRS
III subscore). This is in line with the UPDRS improvements with rotigotine versus placebo in the full study population [20-22]. The improvements in UPDRS scores following rotigotine add-on were not a result of large improvements in a few patients, as the UPDRS responder analysis indicated that approximately twice the proportion of patients treated with combination therapy than patients treated with selegiline monotherapy experienced at least a $20 \%$ improvement in their UPDRS II+III scores. These results are similar to those observed in a randomized, double-blind, placebo-controlled 6-month study of ropinirole in early-stage PD, where combined selegiline and ropinirole treatment significantly improved UPDRS III scores versus selegiline monotherapy. The study had a similar design to the SP512 and SP513 studies, where selegiline was allowed provided the dose was stable for 4 weeks prior to the study and for the duration of the study [24]. Altogether, the results of the current post hoc analyses and the ropinirole study suggest that the addition of a DA to an existing MAO-B inhibitor treatment may improve PD symptoms, in particular motor control.

In the current post hoc analyses, younger patients appeared to benefit more from the concomitant treatment with rotigotine and selegiline than the older patients, as observed by greater treatment differences in the absolute UPDRS scores, and slightly numerically larger improvements in the percentage change of the mean UPDRS scores in the younger patient group ( $\leq 65$ years) versus the older patient group ( $>65$ years). According to the European Federation of Neurological Societies and the Movement Disorder Society (European Section), the recommended initial adjustment of MAO-B inhibitor monotherapy in early-stage PD is to add a DA in younger patients (more likely to develop motor complications) and to add levodopa in older patients (less prone to motor complications) [1]. The low patient number in the older patient subgroup (44 patients vs 86 patients in the younger patient subgroup) limit the conclusions that can be made for older patients; however, our results are in support of the addition of rotigotine to MAO-B inhibitor treatment for greater improvement of PD symptoms in younger patients, and also suggest that improvements with rotigotine and MAO$\mathrm{B}$ inhibitor combination therapy may be achieved in older patients.

The AE profile of rotigotine add-on to patients already receiving selegiline was consistent with dopaminergic stimulation and the use of a transdermal 
Table 2

Incidence of adverse events occurring in $\geq 5 \%$ in any treatment group (safety set)

\begin{tabular}{lcc}
\hline Preferred term & Selegiline+Placebo $(n=46)$ & Selegiline+Rotigotine $(n=84)$ \\
\hline Any adverse event & $42(91)$ & $71(85)$ \\
Application site reaction & $7(15)$ & $35(42)$ \\
Nausea & $9(20)$ & $22(26)$ \\
Somnolence & $7(15)$ & $22(26)$ \\
Dizziness & $3(7)$ & $14(17)$ \\
Insomnia & $3(7)$ & $5(6)$ \\
Upper respiratory tract infection & $3(7)$ & $5(6)$ \\
Constipation & $3(7)$ & $4(5)$ \\
Back pain & $2(4)$ & $6(7)$ \\
Hypertension & $2(4)$ & $4(5)$ \\
Fall & $2(4)$ & $4(5)$ \\
Myalgia & $3(7)$ & $3(4)$ \\
Coughing & $3(7)$ & $2(2)$ \\
Vomiting & 0 & $6(7)$ \\
Headache & 0 & $6(7)$ \\
Pruritus & $1(2)$ & $4(5)$ \\
Dry mouth & 0 & $6(7)$ \\
Peripheral edema & 0 & $5(6)$ \\
Rash & 0 & $4(5)$ \\
\hline
\end{tabular}

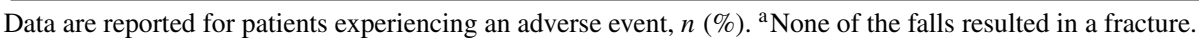

patch. Most common AEs were ASRs, nausea, somnolence and dizziness. The same AEs were reported as most common for the full study population (including patients not receiving selegiline) in the SP512 and SP513 studies [20-22]. Orthostatic hypotension is a common non-motor symptom in $\mathrm{PD}$, and is thought to be caused by both cardiac and extra-cardiac noradrenergic denervation and arterial baroreflex failure [25]. In addition, PD medication, including selegiline and DAs, can increase the frequency of this symptom, and orthostatic hypotension should be specifically considered when new medication is started or added to an existing PD treatment [23]. In these post hoc analyses, only a few patients reported orthostatic hypotension as AEs in both groups; however, vital sign data suggested less patients experienced orthostatic hypotension in the combination therapy group ("Selegiline+Rotigotine") vs selegiline alone. As DAs have not been shown to have a beneficial effect on orthostatic hypotension [23], these data do not reflect the current understanding of the impact of DAs on orthostatic hypotension in PD; the unusual vital sign data may reflect the exploratory nature of this analysis. However, the current observations at least suggest that supplementing existing selegiline treatment with rotigotine is unlikely to lead to an increase in the incidence of orthostatic hypotension.

The addition of rotigotine in patients receiving an MAO-B inhibitor may potentially delay the initiation of treatment with levodopa and thus levodopaassociated motor complications. Whilst long-term open-label extensions of these double-blind studies were performed $[26,27]$, we were unable to evaluate the long-term effect of rotigotine add-on to selegiline, including any levodopa-sparing effect, owing to (1) the small sample of patients receiving selegiline who proceeded to participate in the open-label studies, and (2) the fact that the dose of selegiline could be adjusted in the open-label studies (or indeed an MAO-B inhibitor could be initiated for the first time).

These analyses have several limitations. Firstly, as post hoc, all analyses were performed in an exploratory manner only; therefore, interpretation of the data and conclusions should be made with caution. Secondly, the ability to generalize the results of these post hoc analyses is restricted by the entry criteria of the original double-blind studies, and so limited to patients with early-stage PD of up to 5 years duration and not receiving levodopa. Thirdly, the analyses could be performed only for patients on selegiline, as the MAO-B inhibitor rasagiline was not yet available at the time. It is therefore difficult to ascertain whether adding rotigotine to rasagiline may result in similar benefits as those observed with the combination of rotigotine and selegiline. In addition, selegiline and rasagiline have not been compared in head-to-head studies in early-PD, and literature indirectly comparing the efficacy of the two MAO-B inhibitors is inconclusive $[28,29]$. Finally, it is worth noting the $20 \%$ difference in the gender distribution between the two groups. Gender differences in PD have been recognized, including differences in symptom 
characteristics [30], disease course [31] and PD treatment response [32].

\section{Conclusions}

Despite the limitations of the current analyses, the results support adjunctive treatment with rotigotine in patients already receiving an MAO-B inhibitor for greater improvement in UPDRS II+III total score; the combined therapy may be particularly beneficial for the control of motor signs of PD.

\section{ACKNOWLEDGEMENTS INCLUDING SOURCES OF SUPPORT}

This post hoc analysis was supported by UCB Pharma, Monheim am Rhein, Germany. The authors thank the patients and their caregivers in addition to the investigators and their teams who contributed to the studies included in these analyses. The authors also acknowledge Karolina Rzeniewicz, PhD (Evidence Scientific Solutions, London, UK) for writing and editorial assistance, which was funded by UCB Pharma, and Jesse Fishman, PharmD (UCB Pharma, Smyrna, GA, USA) for publication coordination. The writing and editorial assistance included drafting of the manuscript outline, based on input provided by the authors at a manuscript initiation meeting, and was followed by the coordination of the author review process, incorporation of author comments, editing and formatting the text, production and formatting of figures, verifying the accuracy of the data and references, and collecting author conflict of interest statements. The authors also acknowledge Elisabeth Dohin, MD (UCB Pharma, Brussels, Belgium) for scientific and medical input into the data analyses and interpretation, and Marc Derycke, MSc (UCB Pharma, Monheim am Rhein, Germany) for his contributions to the specifications and programming of the post hoc analyses.

\section{CONFLICT OF INTEREST}

Nir Giladi serves as a member of the Editorial Board for the Journal of Parkinson's Disease, and as a consultant to Teva-Lundbeck, IntecPharma, NeuroDerm, Armon Neuromedical Ltd $\backslash$ Dexel, Monfort, Pharma 2B, and Lysosomal Therapeutic Inc., provided expert testimony to GSK, served on advisory boards to Teva-Lundbeck, Lysosomal Therapeutics, Dexel, Abviee, NeuroDerm, and Sionara, received honoraria from Teva-Lundbeck, Novartis,
UCB Pharma, Movement Disorders Society, Genzyme and Sheir, received payments for lectures at Teva-Lundbeck, Novartis, UCB Pharma, Abviee, Shaier and Genzyme, received research support from the Michael J Fox Foundation, the National Parkinson Foundation, the European Union 7th Framework Program, the Israel Science Foundation, Teva NNE program, Lysosomal Therapeutics, and Abviee, and owns stocks in Lysosomal Therapeutic Inc.; Mahnaz Asgharnejad, Lars Bauer, Frank Grieger and Babak Boroojerdi are salaried employees of UCB Pharma, and receive stock options from their employment.

\section{REFERENCES}

[1] Oertel WH BA, Bloem BR, Bonuccelli U, Burn D, Deuschl G, Dietrichs E, Fabbrini G, Ferreira JJ, Friedman A, Kanovsky P, Kostic V, Nieuwboer A, Odin P, Poewe W, Rascol O, Sampaio C, Schupbach M, Tolosa E, \& Trenkwalder C (2011) Early (uncomplicated) Parkinson's disease. In European Handbook of Neurological Management, Gilhus NE, Barnes MP, Brainin M, eds. Blackwell Publishing Ltd., pp. 217-236.

[2] Connolly BS, \& Lang AE (2014) Pharmacological treatment of Parkinson disease: A review. JAMA, 311, 1670-1683.

[3] Stocchi F, Vacca L, \& Radicati FG (2015) How to optimize the treatment of early stage Parkinson's disease. Transl Neurodegener, $\mathbf{4}, 4$.

[4] Ferreira JJ, Katzenschlager R, Bloem BR, Bonuccelli U, Burn D, Deuschl G, Dietrichs E, Fabbrini G, Friedman A, Kanovsky P, Kostic V, Nieuwboer A, Odin P, Poewe W, Rascol O, Sampaio C, Schupbach M, Tolosa E, Trenkwalder C, Schapira A, Berardelli A, \& Oertel WH (2013) Summary of the recommendations of the EFNS/MDS-ES review on therapeutic management of Parkinson's disease. Eur J Neurol, 20, 5-15.

[5] Macleod AD, Counsell CE, Ives N, \& Stowe R (2005) Monoamine oxidase B inhibitors for early Parkinson's disease. Cochrane Database Syst Rev, CD004898.

[6] Ives NJ, Stowe RL, Marro J, Counsell C, Macleod A, Clarke CE, Gray R, \& Wheatley K (2004) Monoamine oxidase type $\mathrm{B}$ inhibitors in early Parkinson's disease: Meta-analysis of 17 randomised trials involving 3525 patients. BMJ, 329, 593.

[7] Caslake R, Macleod A, Ives N, Stowe R, \& Counsell C (2009) Monoamine oxidase B inhibitors versus other dopaminergic agents in early Parkinson's disease. Cochrane Database Syst Rev, CD006661.

[8] PD MED Collaborative Group (2014) Long-term effectiveness of dopamine agonists and monoamine oxidase B inhibitors compared with levodopa as initial treatment for Parkinson's disease (PD MED): A large, open-label, pragmatic randomised trial. Lancet, 384, 1196-1205.

[9] Pahwa R, \& Lyons KE (2014) Treatment of early Parkinson's disease. Curr Opin Neurol, 27, 442-449.

[10] Löhle M, \& Reichmann H (2011) Controversies in neurology: Why monoamine oxidase B inhibitors could be a good choice for the initial treatment of Parkinson's disease. BMC Neurol, 11, 112.

[11] Schapira AH (2011) Monoamine oxidase B inhibitors for the treatment of Parkinson's disease: A review of symptomatic 
and potential disease-modifying effects. CNS Drugs, 25, 1061-1071.

[12] The Parkinson Study Group (1989) Effect of deprenyl on the progression of disability in early Parkinson's disease. The Parkinson Study Group. N Engl J Med, 321, 1364-1371.

[13] Myllylä VV, Sotaniemi KA, Vuorinen JA, \& Heinonen EH (1992) Selegiline as initial treatment in de novo parkinsonian patients. Neurology, 42, 339-343.

[14] Pålhagen S, Heinonen EH, Hägglund J, Kaugesaar T, Kontants H, Mäki-Ikola O, Palm R, \& Turunen J (1998) Selegiline delays the onset of disability in de novo parkinsonian patients. Swedish Parkinson Study Group. Neurology, 51, 520-525.

[15] Teräväinen H (1990) Selegiline in Parkinson's disease. Acta Neurol Scand, 81, 333-336.

[16] Allain H, Pollak P, \& Neukirch HC (1993) Symptomatic effect of selegiline in de novo Parkinsonian patients. The French Selegiline Multicenter Trial. Mov Disord, 8 (Suppl 1), S36-40.

[17] Mally J, Kovacs AB, \& Stone TW (1995) Delayed development of symptomatic improvement by deprenyl in Parkinson's disease. J Neurol Sci, 134, 143-145.

[18] Scheller D, Ullmer C, Berkels R, Gwarek M, \& Lubbert $\mathrm{H}$ (2009) The in vitro receptor profile of rotigotine: A new agent for the treatment of Parkinson's disease. Naunyn Schmiedebergs Arch Pharmacol, 379, 73-86.

[19] Elshoff JP, Braun M, Andreas JO, Middle M, \& Cawello W (2012) Steady-state plasma concentration profile of transdermal rotigotine: An integrated analysis of three, open-label, randomized, phase I multiple dose studies. Clin Ther, 34, 966-978.

[20] Giladi N, Boroojerdi B, Korczyn AD, Burn DJ, Clarke CE, \& Schapira AH (2007) Rotigotine transdermal patch in early Parkinson's disease: A randomized, double-blind, controlled study versus placebo and ropinirole. Mov Disord, 22, 2398-2404.

[21] Jankovic J, Watts RL, Martin W, \& Boroojerdi B (2007) Transdermal rotigotine: Double-blind, placebo-controlled trial in Parkinson disease. Arch Neurol, 64, 676-682.

[22] Watts RL, Jankovic J, Waters C, Rajput A, Boroojerdi B, \& Rao J (2007) Randomized, blind, controlled trial of trans- dermal rotigotine in early Parkinson disease. Neurology, 68, 272-276.

[23] Sanchez-Ferro A, Benito-Leon J, \& Gomez-Esteban JC (2013) The management of orthostatic hypotension in Parkinson's disease. Front Neurol, 4, 64.

[24] Adler CH, Sethi KD, Hauser RA, Davis TL, Hammerstad JP, Bertoni J, Taylor RL, Sanchez-Ramos J, \& O’Brien CF (1997) Ropinirole for the treatment of early Parkinson's disease. The Ropinirole Study Group. Neurology, 49, 393-399.

[25] Fereshtehnejad SM, \& Lökk J (2014) Orthostatic hypotension in patients with Parkinson's disease and atypical parkinsonism. Parkinsons Dis, 2014, 475854.

[26] Elmer LW, Surmann E, Boroojerdi B, \& Jankovic J (2012) Long-term safety and tolerability of rotigotine transdermal system in patients with early-stage idiopathic Parkinson's disease: A prospective, open-label extension study. Parkinsonism Relat Disord, 18, 488-493.

[27] Giladi N, Boroojerdi B, \& Surmann E (2013) The safety and tolerability of rotigotine transdermal system over a 6year period in patients with early-stage Parkinson's disease. J Neural Transm (Vienna), 120, 1321-1329.

[28] Marconi S, \& Zwingers T (2014) Comparative efficacy of selegiline versus rasagiline in the treatment of early Parkinson's disease. Eur Rev Med Pharmacol Sci, 18, 1879-1882.

[29] Jost WH, Friede M, \& Schnitker J (2012) Indirect metaanalysis of randomised placebo-controlled clinical trials on rasagiline and selegiline in the symptomatic treatment of Parkinson's disease. Basal Ganglia, 2, S17-S26.

[30] Szewczyk-Krolikowski K, Tomlinson P, Nithi K, WadeMartins R, Talbot K, Ben-Shlomo Y, \& Hu MT (2014) The influence of age and gender on motor and non-motor features of early Parkinson's disease: Initial findings from the Oxford Parkinson Disease Center (OPDC) discovery cohort. Parkinsonism Relat Disord, 20, 99-105.

[31] Chillag-Talmor O, Giladi N, Linn S, Gurevich T, El-Ad B, Silverman B, Friedman N, \& Peretz C (2013) Estimation of Parkinson's disease survival in Israeli men and women, using health maintenance organization pharmacy data in a unique approach. $J$ Neurol, 260, 62-70.

[32] Shulman LM (2007) Gender differences in Parkinson's disease. Gend Med, 4, 8-18. 